Bull. Austral. Math. Soc.

VoL. 50 (1994) [117-121]

\title{
Á SIMPLE PROOF OF AN IDENTITY OF PETHE AND HORADAM
}

\section{KUO-JYE CHEN}

An identity for two generalised Tribonacci sequences is obtained. Earlier identities by Pethe and Horadam follow as special cases.

\section{INTRODUCTION}

In 1986, Pethe and Horadam [2] extended Harman's idea [1] and proved

$$
\begin{aligned}
p_{2} \sum_{j=1}^{k}\left(q_{1} q_{2}\right)^{k-j} U_{m+2 j+s} V_{n+2 j+s} & -p_{1} q_{2} \sum_{j=1}^{k}\left(q_{1} q_{2}\right)^{k-j} U_{m+2 j-1+s} V_{n+2 j-1+s} \\
& =U_{m+2 k+s} V_{n+2 k+1+s}-\left(q_{1} q_{2}\right)^{k} U_{m+s} V_{n+1+s}
\end{aligned}
$$

where $U_{n+2}=p_{1} U_{n+1}-q_{1} U_{n}, V_{n+2}=p_{2} V_{n+1}-q_{2} V_{n}, p_{1}, p_{2}, q_{1}$ and $q_{2}$ are fixed real numbers, $s=0$ if $k$ is even, and $s=1$ if $\mathrm{k}$ is odd.

Using (1), they "obtained a wealth of significant summation identities involving the products of combinations of Fibonacci numbers and polynomials, Pell numbers and polynomials, Chebyshev polynomials and sine functions".

The object of this paper is to show that (1) can be proved without using Harman's idea and that (1) is indeed an easy consequence of a very simple reduction formula (see Lemma 1).

We shall use Lemma 1 to obtain an identity (see Theorem 1) for generalised Tribonacci sequences, of which (1) is a special case. Also from Theorem 1 we obtain as a bonus the main result in Pethe's paper [3], that is,

$$
\begin{gathered}
P \sum_{j=1}^{k} Q^{k-j} J_{m+j} J_{n+j}+R\left[\sum_{j=1}^{[k / 2]+s} Q^{k-2 j+s} J_{m+2 j-s} J_{n+2 j-2-s}\right. \\
+\sum_{j=1}^{[k / 2]} Q^{k-2 j+1-s} J_{m+2 j-3+s} J_{n+2 j-1+\bullet} \\
=J_{m+k} J_{n+k+1}-Q^{k} J_{m+s} J_{n+1-s},
\end{gathered}
$$

where $J_{n+3}=P J_{n+2}+Q J_{n+1}+R J_{n}$, and $P, Q, R$ are fixed real numbers.

Received 5th October, 1993.

Copyright Clearance Centre, Inc. Serial-fee code: 0004-9729/94 \$A2.00+0.00. 


\section{The Main Result}

LEMMA 1. Let $\left(U_{n}\right)_{n=0}^{\infty}$ and $\left(V_{n}\right)_{n=0}^{\infty}$ be two sequences satisfying

$$
\text { and } \begin{array}{ll}
U_{n+3} & =p_{1} U_{n+2}+q_{1} U_{n+1}+r_{1} U_{n} \\
V_{n+3} & =p_{2} V_{n+2}+q_{2} V_{n+1}+r_{2} V_{n},
\end{array}
$$

where $p_{i}, q_{i}, r_{i}, i=1,2$, are fixed real numbers (or polynomials). Then

(3) $U_{m+k} V_{n+k+1}=\left(p_{2} U_{m+k} V_{n+k}+p_{1} q_{2} U_{m+k-1} V_{n+k-1}+r_{2} U_{m+k} V_{n+k-2}\right.$

$$
\left.+r_{1} q_{2} U_{m+k-3} V_{n+k-1}\right)+q_{1} q_{2} U_{m+k-2} V_{n+k-1} \text {. }
$$

Proof: By the recurrences satisfied by $U_{n}$ and $V_{n}$, we have

$$
\begin{aligned}
U_{m+k} V_{n+k+1}= & U_{m+k}\left(p_{2} V_{n+k}+q_{2} V_{n+k-1}+r_{2} V_{n+k-2}\right) \\
= & p_{2} U_{m+k} V_{n+k}+r_{2} U_{m+k} V_{n+k-2}+q_{2} U_{m+k} V_{n+k-1} \\
= & p_{2} U_{m+k} V_{n+k}+r_{2} U_{m+k} V_{n+k-2} \\
& \quad+q_{2}\left(p_{1} U_{m+k-1}+q_{1} U_{m+k-2}+r_{1} U_{m+k-3}\right) V_{n+k-1} \\
= & \left(p_{2} U_{m+k} V_{n+k}+p_{1} q_{2} U_{m+k-1} V_{n+k-1}+r_{2} U_{m+k} V_{n+k-2}\right. \\
& \left.\quad+r_{1} q_{2} U_{m+k-3} V_{n+k-1}\right)+q_{1} q_{2} U_{m+k-2} V_{n+k-1} .
\end{aligned}
$$

THEOREM 1. Let $U_{n}$ and $V_{n}$ be as defined in Lemma 1. Then

(4)

$$
\begin{aligned}
p_{2} \sum_{j=1}^{\lfloor k / 2\rfloor}\left(q_{1} q_{2}\right)^{\lfloor k / 2\rfloor-j} U_{m+2 j+.} V_{n+2 j+s} & +p_{1} q_{2} \sum_{j=1}^{\lfloor k / 2\rfloor}\left(q_{1} q_{2}\right)^{\lfloor k / 2\rfloor-j} U_{m+2 j-1+,} V_{n+2 j-1+s} \\
& +r_{2} \sum_{j=1}^{\lfloor k / 2\rfloor}\left(q_{1} q_{2}\right)^{\lfloor k / 2\rfloor-j} U_{m+2 j+,} V_{n+2 j-2+,} \\
& +r_{1} q_{2} \sum_{j=1}^{\lfloor k / 2\rfloor}\left(q_{1} q_{2}\right)^{\lfloor k / 2\rfloor-j} U_{m+2 j-3+,} V_{n+2 j-1+\bullet} \\
& =U_{m+k} V_{n+k+1}-\left(q_{1} q_{2}\right)^{\lfloor k / 2\rfloor} U_{m+,}, V_{n+1+\bullet},
\end{aligned}
$$


or equivalently

(5)

$$
\begin{aligned}
p_{2} \sum_{j=1}^{\lfloor k / 2\rfloor+s}\left(q_{1} q_{2}\right)^{\lfloor k / 2\rfloor-j+s} U_{m+2 j-s} V_{n+2 j-s} & +p_{1} q_{2} \sum_{j=1}^{\lfloor k / 2\rfloor}\left(q_{1} q_{2}\right)^{\lfloor k / 2\rfloor-j} U_{m+2 j-1+s} V_{n+2 j-1+s} \\
& +r_{2} \sum_{j=1}^{\lfloor k / 2\rfloor+s}\left(q_{1} q_{2}\right)^{\lfloor k / 2\rfloor-j+s} U_{m+2 j-s} V_{n+2 j-2-s} \\
& +r_{1} q_{2} \sum_{j=1}^{\lfloor k / 2\rfloor}\left(q_{1} q_{2}\right)^{\lfloor k / 2\rfloor-j} U_{m+2 j-3+s} V_{n+2 j-1+s} \\
& =U_{m+k} V_{n+k+1}-q_{2}^{s}\left(q_{1} q_{2}\right)^{\lfloor k / 2\rfloor} U_{m+s} V_{n+1-s}
\end{aligned}
$$

where $s=0$ if $k$ is even and $s=1$ if $k$ is odd.

ProOF: When $k$ is even, repeated use of (3) yields

$$
\begin{aligned}
U_{m+k} V_{n+k+1}=\left(p_{2}\right. & U_{m+k} V_{n+k}+p_{1} q_{2} U_{m+k-1} V_{n+k-1} \\
+ & \left.r_{2} U_{m+k} V_{n+k-2}+r_{1} q_{2} U_{m+k-3} V_{n+k-1}\right) \\
+ & q_{1} q_{2}\left(p_{2} U_{m+k-2} V_{n+k-2}+p_{1} q_{2} U_{m+k-3} V_{n+k-3}\right. \\
& \left.+r_{2} U_{m+k-2} V_{n+k-4}+r_{1} q_{2} U_{m+k-5} V_{n+k-3}\right) \\
+ & \cdots \\
+ & \left(q_{1} q_{2}\right)^{(k-4) / 2}\left(p_{2} U_{m+4} V_{n+4}+p_{1} q_{2} U_{m+3} V_{n+3}\right. \\
& \left.+r_{2} U_{m+4} V_{n+2}+r_{1} q_{2} U_{m+1} V_{n+3}\right) \\
+ & \left(q_{1} q_{2}\right)^{(k-2) / 2}\left(p_{2} U_{m+2} V_{n+2}+p_{1} q_{2} U_{m+1} V_{n+1}\right. \\
& \left.+r_{2} U_{m+2} V_{n}+r_{1} q_{2} U_{m-1} V_{n+1}\right) \\
& +\left(q_{1} q_{2}\right)^{k / 2} U_{m} V_{n+1}
\end{aligned}
$$

whence

(6)

$$
\begin{gathered}
p_{2} \sum_{j=1}^{k / 2}\left(q_{1} q_{2}\right)^{(k / 2)-j} U_{m+2 j} V_{n+2 j}+p_{1} q_{2} \sum_{j=1}^{k / 2}\left(q_{1} q_{2}\right)^{(k / 2)-j} U_{m+2 j-1} V_{n+2 j-1} \\
+r_{2} \sum_{j=1}^{k / 2}\left(q_{1} q_{2}\right)^{(k / 2)-j} U_{m+2 j} V_{n+2 j-2}+r_{1} q_{2} \sum_{j=1}^{k / 2}\left(q_{1} q_{2}\right)^{(k / 2)-j} U_{m+2 j-3} V_{n+2 j-1} \\
=U_{m+k} V_{n+k+1}-\left(q_{1} q_{2}\right)^{k / 2} U_{m} V_{n+1}, \quad k \text { even. }
\end{gathered}
$$


Similarly, when $k$ is odd, we have

$$
\begin{aligned}
U_{m+k} V_{n+k+1}= & \left(p_{2} U_{m+k} V_{n+k}+p_{1} q_{2} U_{m+k-1} V_{n+k-1}\right. \\
+ & \left.r_{2} U_{m+k} V_{n+k-2}+r_{1} q_{2} U_{m+k-3} V_{n+k-1}\right) \\
+ & q_{1} q_{2}\left(p_{2} U_{m+k-2} V_{n+k-2}+p_{1} q_{2} U_{m+k-3} V_{n+k-3}\right. \\
& \left.+r_{2} U_{m+k-2} V_{n+k-4}+r_{1} q_{2} U_{m+k-5} V_{n+k-3}\right) \\
+ & \cdots \\
+ & \left(q_{1} q_{2}\right)^{(k-5) / 2}\left(p_{2} U_{m+5} V_{n+5}+p_{1} q_{2} U_{m+4} V_{n+4}\right. \\
& \left.+r_{2} U_{m+5} V_{n+3}+r_{1} q_{2} U_{m+2} V_{n+4}\right) \\
+ & \left(q_{1} q_{2}\right)^{(k-3) / 2}\left(p_{2} U_{m+3} V_{n+3}+p_{1} q_{2} U_{m+2} V_{n+2}\right. \\
& \left.+r_{2} U_{m+3} V_{n+1}+r_{1} q_{2} U_{m} V_{n+2}\right) \\
+ & \left(q_{1} q_{2}\right)^{(k-1) / 2} U_{m+1} V_{n+2}
\end{aligned}
$$

whence

(8)

$$
\begin{gathered}
p_{2} \sum_{j=1}^{(k-1) / 2}\left(q_{1} q_{2}\right)^{(k-1) / 2-j} U_{m+2 j+1} V_{n+2 j+1}+p_{1} q_{2} \sum_{j=1}^{(k-1) / 2}\left(q_{1} q_{2}\right)^{(k-1) / 2-j} U_{m+2 j} V_{n+2 j} \\
+r_{2} \sum_{j=1}^{(k-1) / 2}\left(q_{1} q_{2}\right)^{(k-1) / 2-j} U_{m+2 j+1} V_{n+2 j-1}+r_{1} q_{2} \sum_{j=1}^{(k-1) / 2}\left(q_{1} q_{2}\right)^{(k-1) / 2-j} U_{m+2 j-2} V_{n+2 j} \\
=U_{m+k} V_{n+k+1}-\left(q_{1} q_{2}\right)^{(k-1) / 2} U_{m+1} V_{n+2}, \quad k \text { odd. }
\end{gathered}
$$

Since

$$
\begin{aligned}
U_{m+1} V_{n+2} & =U_{m+1}\left(p_{2} V_{n+1}+q_{2} V_{n}+r_{2} V_{n-1}\right) \\
& =p_{2} U_{m+1} V_{n+1}+q_{2} U_{m+1} V_{n}+r_{2} U_{m+1} V_{n-1},
\end{aligned}
$$

(8) can be rewritten as

(9)

$$
\begin{gathered}
p_{2} \sum_{j=1}^{(k+1) / 2}\left(q_{1} q_{2}\right)^{(k+1) / 2-j} U_{m+2 j-1} V_{n+2 j-1}+p_{1} q_{2} \sum_{j=1}^{(k-1) / 2}\left(q_{1} q_{2}\right)^{(k-1) / 2-j} U_{m+2 j} V_{n+2 j} \\
+r_{2} \sum_{j=1}^{(k+1) / 2}\left(q_{1} q_{2}\right)^{(k+1) / 2-j} U_{m+2 j-1} V_{n+2 j-3}+r_{1} q_{2} \sum_{j=1}^{(k-1) / 2}\left(q_{1} q_{2}\right)^{(k-1) / 2-j} U_{m+2 j-2} V_{n+2 j} \\
=U_{m+k} V_{n+k+1}-q_{2}\left(q_{1} q_{2}\right)^{(k-1) / 2} U_{m+1} V_{n}, \quad k \text { odd. }
\end{gathered}
$$

Now, it is readily seen that (4) follows from (6) and (8), and (5) follows from (6) and (9). 


\section{Special Cases}

In this section, it will be shown, as we proposed in Introduction, that (1) and (2) are special cases of (4) and (5), respectively.

CASE 1. Replacing $k$ first by $2 k$ and then by $2 k+1$ in (4) gives two identities which can be combined in one identity, that is,

$$
\begin{aligned}
p_{2} \sum_{j=1}^{k}\left(q_{1} q_{2}\right)^{k-j} U_{m+2 j+s} V_{n+2 j+s} & +p_{1} q_{2} \sum_{j=1}^{k}\left(q_{1} q_{2}\right)^{k-j} U_{m+2 j-1+s} V_{n+2 j-1+s} \\
& +r_{2} \sum_{j=1}^{k}\left(q_{1} q_{2}\right)^{k-j} U_{m+2 j+s} V_{n+2 j-2+s} \\
& +r_{1} q_{2} \sum_{j=1}^{k}\left(q_{1} q_{2}\right)^{k-j} U_{m+2 j-3+s} V_{n+2 j-1+s} \\
& =U_{m+2 k+s} V_{n+2 k+1+s}-\left(q_{1} q_{2}\right)^{k} U_{m+s} V_{n+1+s}
\end{aligned}
$$

Let $q_{1} \rightarrow-q_{1}, q_{2} \rightarrow-q_{2}$, and $r_{1}=r_{2}=0$. Then (10) reduces to (1).

CASE 2. Let $U_{n}=V_{n}=J_{n}, p_{1}=p_{2}=P, q_{1}=q_{2}=Q$, and $r_{1}=r_{2}=R$. Then we obtain (2) from (5) by combining even and odd cases of $k$.

\section{References}

[1] C.J. Harman, 'Complex Fibonacci numbers', Fibonacci Quart. 19 (1981), 82-86.

[2] S. Pethe and A.F. Horadam, 'Generalized Gaussian Fibonacci numbers', Bull. Austral. Math. Soc. 33 (1986), 37-48.

[3] S. Pethe, 'Some identities for Tribonacci sequences', The Fibonacci quarterly 26 (1988), 144-151.

Department of Mathematics

Changhua University of Education

Changhua 500

Taiwan 